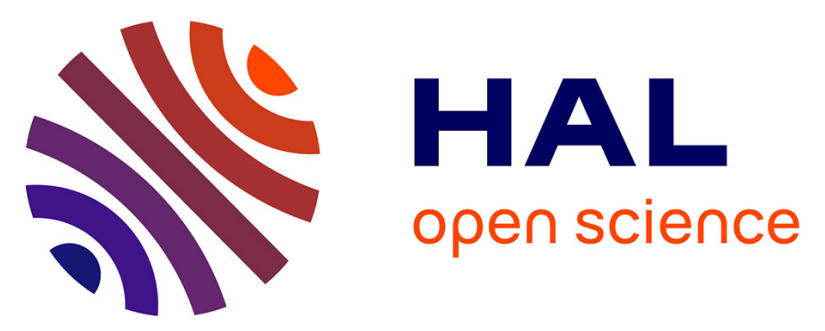

\title{
Superconductivity and absence of a Kohn anomaly in the quasi-one-dimensional organic conductor : (TMTSF)2AsF6
}

M. Ribault, J.-P. Pouget, D. Jérome, K. Bechgaard

\section{- To cite this version:}

M. Ribault, J.-P. Pouget, D. Jérome, K. Bechgaard. Superconductivity and absence of a Kohn anomaly in the quasi-one-dimensional organic conductor: (TMTSF)2AsF6. Journal de Physique Lettres, 1980, 41 (24), pp.607-610. 10.1051/jphyslet:019800041024060700 . jpa-00231857

\section{HAL Id: jpa-00231857 https://hal.science/jpa-00231857}

Submitted on 1 Jan 1980

HAL is a multi-disciplinary open access archive for the deposit and dissemination of scientific research documents, whether they are published or not. The documents may come from teaching and research institutions in France or abroad, or from public or private research centers.
L'archive ouverte pluridisciplinaire HAL, est destinée au dépôt et à la diffusion de documents scientifiques de niveau recherche, publiés ou non, émanant des établissements d'enseignement et de recherche français ou étrangers, des laboratoires publics ou privés. 


\title{
Superconductivity and absence of a Kohn anomaly in the quasi-one-dimensional organic conductor : $\left(\mathrm{TMTSF}_{2} \mathbf{A s F}_{6}\left({ }^{+}\right)\right.$
}

\author{
M. Ribault, J.-P. Pouget, D. Jérome \\ Laboratoire de Physique des Solides (*), Université Paris-Sud, 91405 Orsay, France \\ and K. Bechgaard \\ H. C. Oersted Institute, Universitetsparken 5, DK-2100, Copenhagen, Denmark
}

(Reçule 3 octobre 1980, acceptéle 22 octobre 1980)

\begin{abstract}
Résumé. - Nous avons observé un état supraconducteur dans un second conducteur organique quasi-unidimensionnel : (TMTSF) ${ }_{2} \mathrm{AsF}_{6}$, à la température de $1,1 \mathrm{~K}$ sous $12 \mathrm{kbar}$. L'existence de l'état supraconducteur ne semble apparemment pas reliée à la présence d'un mode mou dans une branche de phonons intermoléculaires, étant donné l'absence d'anomalie de Kohn à $2 k_{\mathrm{F}}$ à basse température et à pression atmosphérique.
\end{abstract}

Abstract. - Superconductivity has been observed in a second quasi-one-dimensional organic conductor : (TMTSF) ${ }_{2} \mathrm{AsF}_{6}$, at a temperature of $1.1 \mathrm{~K}$ under $12 \mathrm{kbar}$. The establishment of the superconducting state is apparently not related to the existence of a soft external phonon mode at $2 k_{\mathrm{F}}$, since under atmospheric pressure $\mathrm{X}$-ray diffuse scattering fails to detect any Kohn anomaly at low temperature.

In this letter we present a set of experiments performed on a new quasi-one-dimensional organic conductor : (TMTSF) ${ }_{2} \mathrm{AsF}_{6}$, (di-2,2'-bi-4.5-dimethyl1.3-diselenole-hexafluoroarsenate).

Resistivity measurements have shown the existence of a superconducting state, stabilized below $1.1 \mathrm{~K}$ at a pressure of 12 kbar. Moreover, X-ray diffuse scattering studies performed at atmospheric pressure show no structural precursor effects at the wave vector $2 k_{\mathrm{F}}$ (Kohn anomaly) in this 1-D conductor at low temperatures. (TMTSF) ${ }_{2} \mathrm{AsF}_{6}$, a salt of the radical cation TMTSF belongs to the family of $2: 1$ stoichiometry salts : $(\mathrm{TMTSF})_{2} \mathrm{X}, \mathrm{X}=\mathrm{PF}_{6}^{-}, \mathrm{AsF}_{6}^{-}, \mathrm{SbF}_{6}^{-}, \mathrm{BF}_{4}^{-}$, $\mathrm{NO}_{3}^{-}$[1]. The triclinic structure of (TMTSF) ${ }_{2} \mathrm{AsF}_{6}$ is isomorphous with that of the conductor (TMTSF) ${ }_{2} \mathrm{PF}_{6}$ in which superconductivity has recently been observed for the first time in an organic compound under pressure $[2,3,4]$. The small singlecrystals used for the present work have been obtained by an electrochemical technique through a constant

$\left({ }^{+}\right)$This letter is the English version of a Note presented to the Academy of Sciences, 29 September 1980.

$\left({ }^{*}\right)$ Laboratoire associé au C.N.R.S. Work supported in part by a DGRST contract $\mathrm{n}^{\circ} 80.7 .0165$. current oxidation of the neutral TMTSF molecule in $\mathrm{CH}_{2} \mathrm{Cl}_{2}$ solution [5].

At ambient conditions the 4-contact conductivity, measured by the usual low frequency techniques amounts to $500 \pm 100(\Omega . \mathrm{cm})^{-1}$. At atmospheric pressure, the conductivity increases as the temperature is decreased [1], but its value at $20 \mathrm{~K}$, $\approx 10^{4}(\Omega . \mathrm{cm})^{-1}$, is smaller (by about one order of magnitude) than the conductivity of (TMTSF) ${ }_{2} \mathrm{PF}_{6}[1]$. A phase transition has been detected by thermopower measurements at $15 \mathrm{~K}$ [1].

The present longitudinal conductivity measurements have been performed under pressure and at very low temperatures with the same equipment used for (TMTSF) ${ }_{2} \mathrm{PF}_{6}$ [2]. In the present case, however, Joule heating effects are seen in samples of small cross section, namely $0.1 \times 0.1 \mathrm{~mm}^{2}$, for currents exceeding $\approx 100 \mu \mathrm{A}$, even though the resistance of the contacts between the lead wires and the organic sample appears to be fairly small ( 2 to $3 \Omega$ per contact). At room temperature $\sigma_{\|}$increases at a rate of $20 \%$ per kbar under pressure. However above 3 kbar microcracks are often seen to develop along the long axis of the crystals.

As shown by the temperature dependence of the 
resistance, a pressure of 12 kbar stabilizes a conducting state (with metallic-like conductivity) down to liquid helium temperature. But once again the maximum conductivity observed at $4.2 \mathrm{~K}$ seems to show an upper limit of $2 \times 10^{4}(\Omega . \mathrm{cm})^{-1}$, whereas values about 10 times larger are currently measured with $(\mathrm{TMTSF})_{2} \mathrm{PF}_{6}[2,6]$.

Several interesting features are revealed by the behaviour of the resistance below $4.2 \mathrm{~K}$ (see figure 1) : (i) a fairly substantial $T$-dependence between 4.2 and $1.4 \mathrm{~K}$, although a stronger dependence is usually observed with (TMTSF) ${ }_{2} \mathrm{PF}_{6}$ [2]; (ii) a transition towards a zero resistance state occurring at $T_{\mathrm{c}}=1.1 \mathrm{~K}$ according to the criterion $R\left(T_{\mathrm{c}}\right)=R(1.4 \mathrm{~K}) / 2$. A similar behaviour has been observed with two samples simultaneously studied with two independent electric circuits.

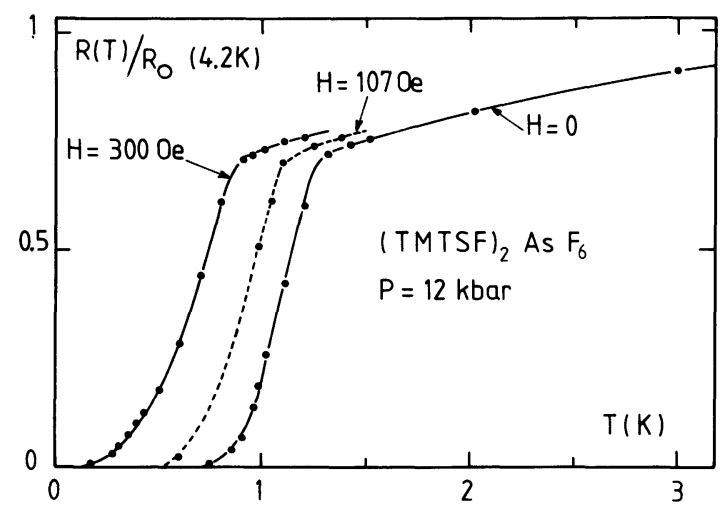

Fig. 1. - Temperature dependence of the resistance of (TMTSF) ${ }_{2} \mathrm{AsF}_{6}$ normalized to its value at $4.2 \mathrm{~K}$ in earth magnetic field (measured with a current of $0.6 \mathrm{~A}$ ). The $4.2 \mathrm{~K}$ sample resistance is $200 \mathrm{~m} \Omega$. The superconducting transition is shifted down in temperature by the application of a transverse magnetic field $(H=107$ and $300 \mathrm{Oe}$ ). As far as the sample shown is concerned, the contact resistance has been removed so as to make the sample resistance vanish at $T=0$.

Application of a magnetic field perpendicular to the chain direction gives unambiguous evidence that a superconducting state exists in (TMTSF) ${ }_{2} \mathrm{AsF}_{6}$ with a critical temperature of $1.1 \mathrm{~K}$ in the earths magnetic field. Besides giving confirmation of the superconductivity phenomenon in hexafluorate pseudo-halogen salts of TMTSF this new discovery leads to some interesting remarks.

On the one hand, the application of a hydrostatic pressure remains a necessary condition for the stabilization of the superconducting state in these organic conductors (a more thorough determination of the (TMTSF) ${ }_{2} \mathrm{AsF}_{6}$ phase diagram is near completion). On the other hand, comparison of electronic properties in the two organic superconductors reveals salient differences. For example, the slope $\mathrm{d} H_{\mathrm{c}}^{\perp} /\left.\mathrm{d} T\right|_{T_{\mathrm{c}}}$ given by $\varphi_{0} / 2 \pi \zeta_{\|}(0) \zeta_{\perp}(0) T_{c}$, within the LandauGinzburg formalism, equals $550 \mathrm{Oe} / \mathrm{K}$ for (TMTSF) ${ }_{2} \mathrm{AsF}_{6}$ as compared with $220 \mathrm{Oe} / \mathrm{K}$ for (TMTSF) ${ }_{2} \mathrm{PF}_{6}$. The substitution of phosphorus for arsenic increases $T_{\mathrm{c}}$ by $20 \%$ at the pressure of $12 \mathrm{kbar}$. Furthermore it looks as if the paraconductivity contribution above $T_{\mathrm{c}}$ is weaker in (TMTSF) ${ }_{2} \mathrm{AsF}_{6}$ than in (TMTSF) ${ }_{2} \mathrm{PF}_{6}$.

Taking into account all these experimental results we feel confident that the newly discovered superconductor (TMTSF) ${ }_{2} \mathrm{AsF}_{6}$ may be less pure and more three-dimensionally coupled than (TMTSF $)_{2} \mathrm{PF}_{6}$.

In order to obtain some understanding of the origin of superconductivity in this organic conductor, X-ray diffuse scattering experiments have been performed in parallel with the above mentioned conductivity studies.

X-ray diffuse scattering experiments have been performed by the fixed film, fixed crystal method with the same experimental set up used for the study of TTF-TCNQ between $20 \mathrm{~K}$ and $300 \mathrm{~K}$ [7]. The principle of the method and its application to the study of one-dimensional (1-D) conductors are given in reference [8]. However note that with this method a large part of reciprocal space can be scanned on a single photograph. For example, about 50 Brillouin zones are included in the pattern shown in figure 2.

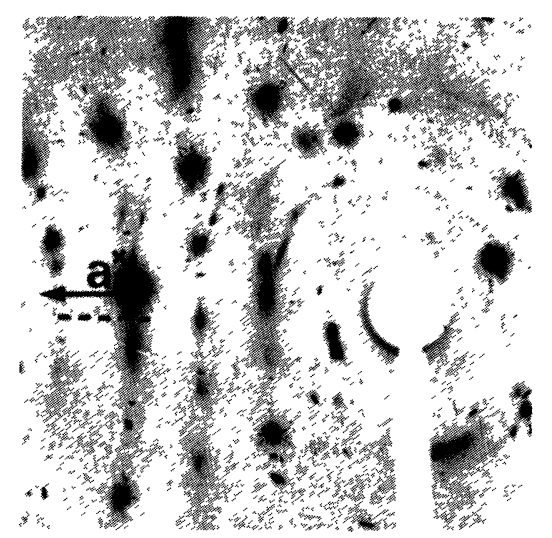

Fig. 2. - X-ray pattern from (TMTSF) ${ }_{2} \mathrm{AsF}_{6}$ at $20 \mathrm{~K}$. The incident radiation is $\mathrm{CuK} \alpha$. The $a^{*}$ reciprocal direction is shown together with the segment scanned (dashed line) in the microdensitometer readings presented figure 3 (the pattern is free from $\lambda / 2$ contamination coming from the incident beam, and the weak spots in rings around the origin are slight desorientations within the sample).

The anion group of (TMTSF) ${ }_{2} \mathrm{AsF}_{6}$ is singly charged $\left(\mathrm{AsF}_{6}^{-}\right)$, leaving $1 / 2$ hole per donor molecule. With a cell containing in chain direction, a zig-zag slightly dimerized of 2 molecules of TMTSF, there is a hole to share between them, leading to a half-filled conduction band, i.e. $2 k_{\mathrm{F}}=a^{*} / 2$. By analogy with previously reported [7-9], X-ray results from 1-D conductors, one excepts to observe, as $2 k_{\mathrm{F}}$ precursors to the structural transitions, diffuse sheets midway between layers of Bragg spots and perpendicular to the chain direction.

Figure 2 shows a monochromatic Laue pattern from (TMTSF) ${ }_{2} \mathrm{AsF}_{6}$ at $20 \mathrm{~K}$. The Bragg spots are surrounded by their usual thermal scattering but no 
supplementary scattering above the continuous diffuse background can be detected. Precursor scatterings have also not been observed between room temperature and $12 \mathrm{~K}$ in $(\mathrm{TMTSF})_{2} \mathrm{PF}_{6}$ [9]. Although in the sulfur analogue of the latter compound, (TMTTF $)_{2} \mathrm{PF}_{6}$, the growth of a quasi-1-D scattering at the expected wave vector $q_{\|}=a^{*} / 2$ [9] has been seen below $60 \mathrm{~K}$.

By comparing studies of the $2 k_{\mathrm{F}}$ anomaly of TTFTCNQ by $X$-ray diffuse scattering and inelastic neutron scattering methods [8], and assuming an inelastic origin for the additional X-ray intensity, one can estimate a threshold of detection of about $5-6 \%$ in the relative drop of phonon frequency, $\Delta \omega / \omega$, at the position of the anomaly, which in this compound is at $150 \mathrm{~K}$. In a compound like (TMTSF $)_{2} \mathrm{AsF}_{6}$ containing Se atoms on the conducting chain which scatter X-rays more strongly, one can, assuming a similar amplitude of vibration, reduce this threshold level to a few percent in the same temperature range.

A study of (TMTSF) ${ }_{2} \mathrm{AsF}_{6}$ has also been made as a function of temperature. Around $100 \mathrm{~K}$, a quasi-1-D scattering at $q_{\|}=0.5 a^{*}$ can just be detected above the thermal scattering (Fig. 3). Its features are reminiscent of that of the $2 k_{\mathrm{F}}$ scattering of TMTSFDMTCNQ at $225 \mathrm{~K}$ where it can first be seen [9]. However in contrast with the $2 k_{\mathrm{F}}$ anomaly of TMTSF-DMTCNQ, there is no significant increase in intensity nor sharpening in width of the weak scattering as the temperature is reduced. The scattering is no longer detectable at $20 \mathrm{~K}$ (Figs. 2 and 3).

But its diffuse appearance, the anomaly observed between about $100 \mathrm{~K}$ and $50 \mathrm{~K}$ is at the extreme limit of sensitivity of our photographic method of detection. Using the estimate given above, it might correspond to a phonon frequency anomaly, $\Delta \omega / \omega$, of a few percent. Its dynamical origin may be confirmed by its absence at low temperatures, where the thermal population factor is unfavourable. However this does not exclude the possibility of a complete disappearance of the phonon anomaly itself at low temperatures.

The latter observation of an extremely weak $2 k_{\mathrm{F}}$ Kohn anomaly indicates the sensitivity of the method of detection used. The fact that this anomaly does not grow appreciably when the temperature is decreased, shows clearly that under atmospheric pressure, (TMTSF) ${ }_{2} \mathrm{AsF}_{6}$ does not undergo a structural instability arising from divergence of the response function related to the formation of charge density waves at the $2 k_{\mathrm{F}}$ wave vector.

Consequently we believe that the absence of a Kohn

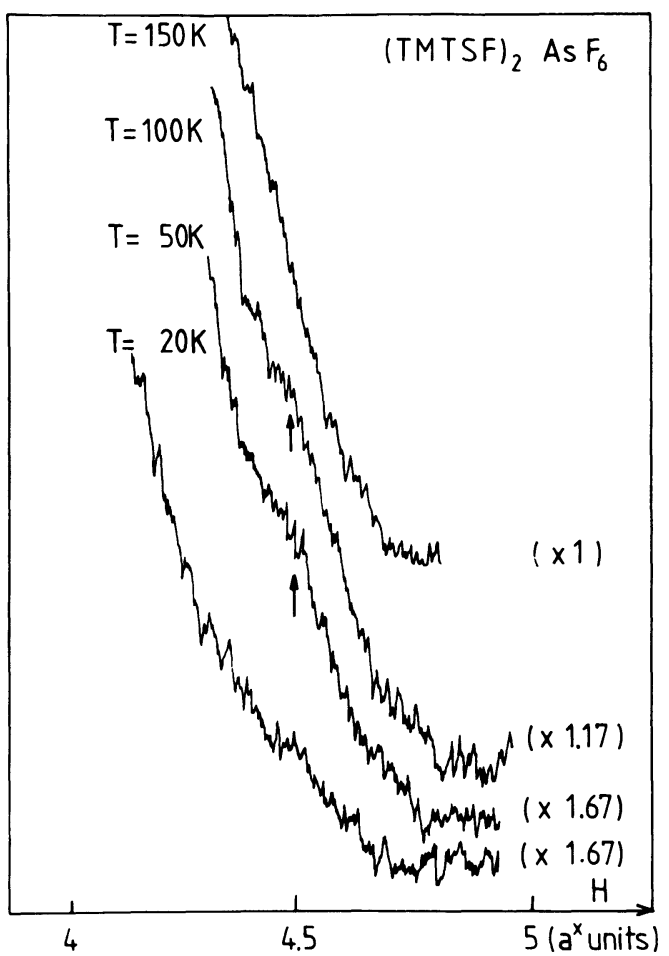

Fig. 3. - Microdensitometer readings along the dashed line shown in figure 2. The readings obtained at $100 \mathrm{~K}$ and $50 \mathrm{~K}$ show a slight increase of the X-ray diffuse intensity (arrow) in the fall of the usual thermal scattering around $h=4.5$ (corresponding to the reduced wave vector $\left.q_{\|}=1 / 2 a^{*}\right)$. The number on the right band are scaling factors related to the exposure time of the $\mathrm{X}$-ray patterns read.

anomaly is also an a fortiori characteristic of (TMTSF $)_{2} \mathrm{AsF}_{6}$ under high pressure $(12 \mathrm{kbar})$, where no metal insulator transition is seen at low temperatures. Finally, the finding of a second organic superconductor strongly suggests that other organic superconductors will be found. The important feature revealed by $\mathrm{X}$-ray diffuse scattering in both (TMTSF) ${ }_{2} \mathrm{AsF}_{6}$ and (TMTSF) ${ }_{2} \mathrm{PF}_{6}$, namely the absence of a low temperature $2 k_{\mathrm{F}}$ Kohn anomaly, leads to the important conclusion that superconductivity in these organic conductors is not related to the existence of a soft external phonon mode at $2 k_{\mathrm{F}}$. Following the above mentioned discussion we consider as very unlikely the situation for which pressure would enhance simultaneously the CDW and superconducting instabilities.

Acknowledgments. - We wish to acknowledge the efficient help given by G. Benedek and D. Mailly in the course of this work, the skilful aid from C. Weyl and very fruitful discussions with $\mathrm{R}$. Comès and $\mathrm{S}$. Megtert.

\section{References}

[1] Bechgaard, K., Jacobsen, C. S., Mortensen, K., Pedersen, H. J. and Thorup, N., Solid State Commun. 33 (1980) 1119.
[2] Jérome, D., Mazaud, A., Ribault, M. and Bechgaard, K., J. Physique Lett. 41 (1980) L-95. 
[3] Ribault, M., Benedek, G., Jérome, D. and Bechgaard, K., J. Physique Lett. 41 (1980) L-397.

[4] Walsh, W. M., Wudl, F., Thomas, G. A., Nalewajek, D., Hauser, J. J., Lee, P. A. and Poehler, T., Phys. Rev. Lett. 45 (1980) 829.

[5] BechGaARD, K., unpublished.

[6] Schulz, H. J., Jérome, D., Mazaud, A., Ribault, M. and BECHGAARD, K., to be published and JÉROME, D. and Schulz, H. J., to be published in Physics in One Dimension, J. Bernasconi editor (Springer) 1980.
[7] Khanna, S. K., Pouget, J. P., Comès, R., Garito, A. F. and Hegger, A. J., Phys. Rev. B 16 (1977) 1468.

[8] Comès, R. and Shirane, G., in Highly Conducting One Dimensional Solids edited by J. T. Devreese, R. P. Evrard and V. E. Van Doren (Plenum Press-New York) 1979, p. 17. [9] Pouget, J. P., to be published in Chemica Scripta (Sweden), Proceedings of the International Conference on Low Dimensional Synthetic Metals, Helsingör, Denmark, August 1980. 